\title{
Design and Implementation of Judge System for Old Man Fall Down
}

\author{
Chao Sun 1, a, Jindong Zhang 1, 2, 3, b, *, Jiajia Lin 1, c, Rui Song 1, d, \\ Xuan Zang ${ }^{2, e}$, Xiaohan Liu ${ }^{2, f}$ \\ ${ }^{1}$ College of Software, Jilin University, Changchun, 130012, China \\ ${ }^{2}$ College of Computer Science and Technology, Jilin University, Changchun, 130012, China \\ ${ }^{3}$ Key Laboratory of Symbol Computation and Knowledge Engineering of the Ministry of Education, \\ Jilin University, Changchun,130012, China \\ a2869341565@qq.com, bzhangjindong_100@163.com, c596706008@qq.com,

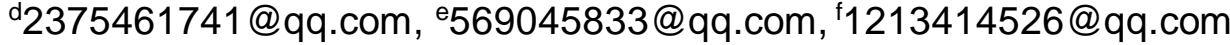

( ${ }^{\star}$ Corresponding author: Jindong Zhang, e-mail: zhangjindong_100@163.com)

Keywords: APP, Fall down, Dynamic models, GPS.

\begin{abstract}
This paper taking into account the universal use of Android phones, designs and implements judgments and notices the old man falling cell phone App. By calling the phones accelerometer, gyroscope collection campaigns after processing according to human body dynamics model to determine the specific state of the elderly, and get the specific latitude and longitude via GPS; Cell phone judge after the old man falls can send text messages to bound phone number in advance and inform the GPS to obtain location coordinates, after the fall in the elderly will be able to make the necessary relief in a timely manner.
\end{abstract}

\section{Introduction}

It is estimated that by 2030, China's aging population will reach 200 million. Along with the empty nest is a series of social problems, such as old man fall down no one dared to help has become a focus of attention. How to ensure the personal safety of empty nest elderly has become a pressing problem. Due to the deteriorating health of the elderly, are prone to falling, and so on, and most of the children of the elderly are busy, not even in the old man, and now the man fell did not receive timely treatment, will seriously affect the health of the elderly life[1].

Facts now community birth had judge wrestling class products, early in 12th session "Challenge Cup" province game works in the on appeared has wear type elderly fell detection guardianship system, its combined currently advanced of communications technology, and sensor technology and mature of signal processing algorithm, in based on mode recognition method of based Shang, according to human three axis acceleration value and single arm heart signals of changes on elderly fell action made judge. Our group study concluded that, after all, wears the device among the elderly people, in many cases mobile phones is also essential, seems a bit redundant, and is not easy to carry [2]. Using Android mobile phone comes with acceleration sensor body acceleration, use fall detection algorithm based on acceleration features the APP's design and implementation, results show that this APP can effectively detect the movement of the elderly fall to determine whether, in the event after stumbling on the old man, send message notification to a contact in a timely manner.

\section{System Description}

This system real-time monitoring elderly phone of acceleration, to this to monitoring elderly time of movement State, judge elderly whether fell, if fell, through GPS gets elderly fell of specific location (first gets longitude degrees, again anti-analytical out specific address), system automatically generated SMS (including elderly of movement State and specific location), sent to, system by bound of contact people (user using Qian himself set good of). The description of system is shown in Fig.1. 

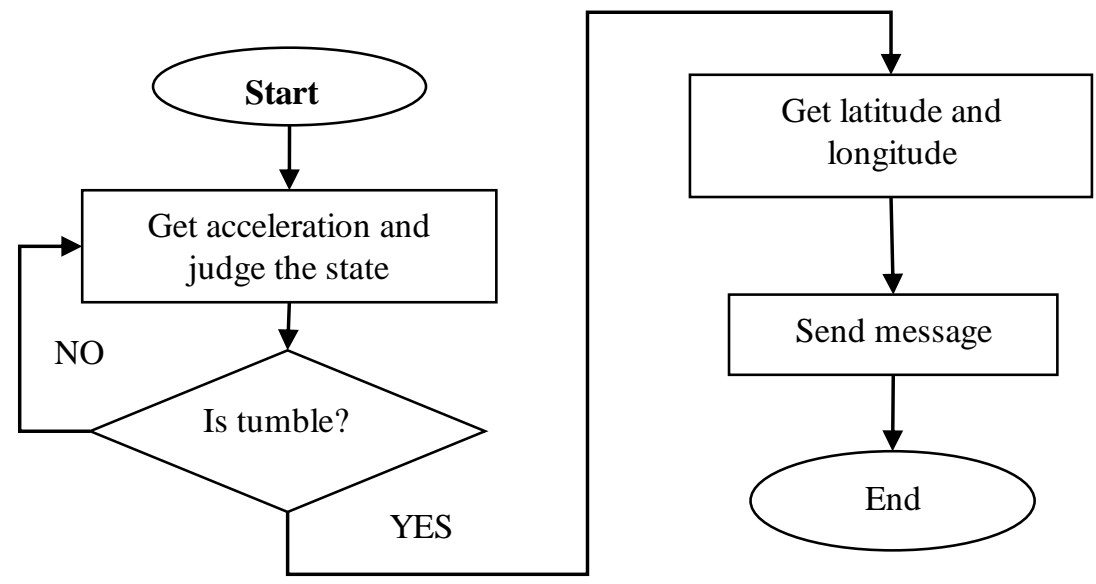

Fig 1. App flow chart

Start App to enable real-time data including through acceleration sensor gets, for angular acceleration gyroscope gets mobile phone GPS to obtain the latitude and longitude information, design an algorithm to obtain the acceleration state judge, judge the data may be judged to fall, fell, according to latitude and longitude to get exact location coordinates, send SMS notification to help families falls location, as shown in Fig2.

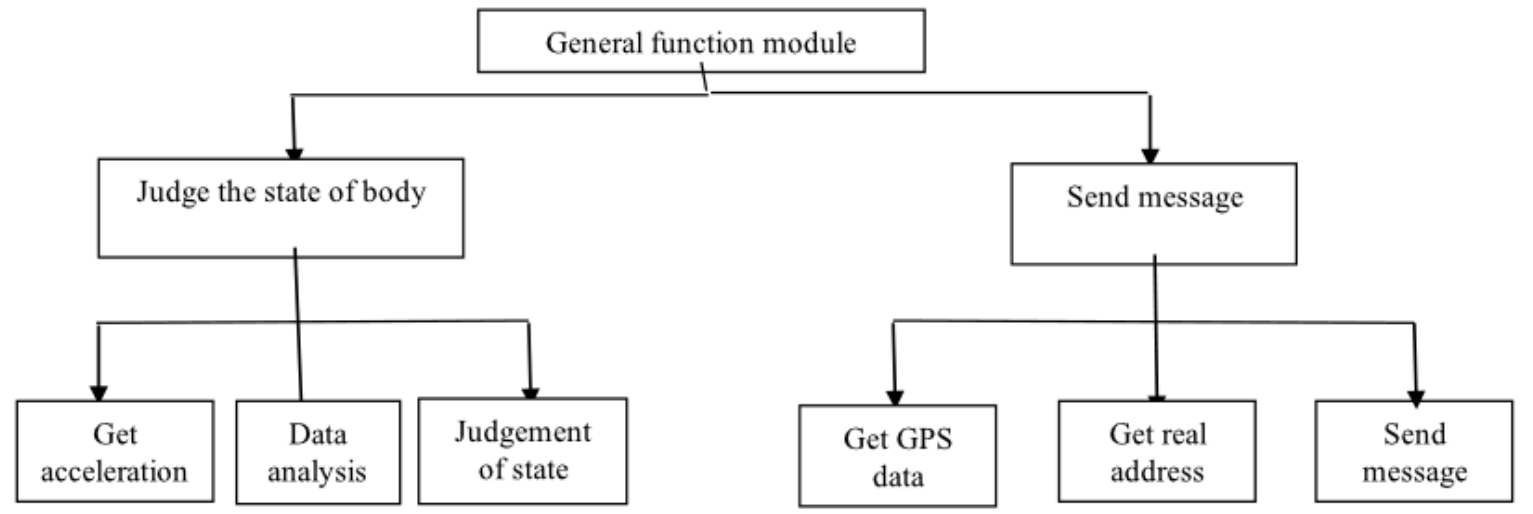

Fig 2. App design module hierarchy graph

In the process of designing app, we're talking about old man fall down the core functionality of the system can be divided into judge fall modules and SMS modules. Judge fell module design method for real-time monitoring of the current state for the elderly, will probably fall to an acceleration of events, acceleration values for data analysis, processing, judge, does not fall the clear information, fell to transmit that information to the SMS module; SMS modules receive the fall message, obtained by the current latitude and longitude information into your location, combined with design message format in the file sent to the old man's family, sending text messages.

\section{Modules Design}

To test whether the elderly fall need to monitor movement of the elderly, and make a judgment, however, walk, run, jump, and activities of daily living or a cell phone thrown will cause acceleration, angular acceleration of change, therefore, we combine human body three dimensional coordinate system data and dynamic models to analyze judgment can accurately determine daily alive or fall.

Certainly acceleration of update is very precise fast of, however for each a speed of changes more du for data processing and then on Human Dynamics model is extremely occupied resources of, at, on need on get of data for simple preliminary of filter, like, vertical ground acceleration change is greater than 2, level direction produced acceleration, and with phone of angular acceleration mutation Shi, on meet has can points new processing into dynamics model than on of data conditions, and usually walk, Running does not record such data, reduces the complexity of space and time. The flow chart in Figure 3: 


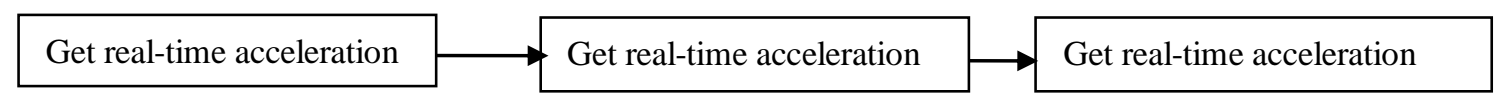

Fig 3. Judge fell module flowchart

The main function of mobile acceleration sensor is to induction the movement of phone. It gets three parameter, that is the result that the acceleration of coordinate axis $\mathrm{x}, \mathrm{y}, \mathrm{z}$ in space coordinate system decrease the component of gravitational acceleration, and the direction is negative direction of gravitation acceleration[3]. Set the ground to fixed Cartesian coordinate system OXYZ, $\mathrm{z}$ axis perpendicular to the ground. Torso coordinate system Oxyz trunk movement and movement, and when the normal vertical coordinate system Oxyz of the body parallel to the coordinate system OXYZ. X axis pointing to the torso forward, pointing to the left $\mathrm{y}$ axis and $\mathrm{z}$ axis pointing to the right. Fall detection algorithm pseudo code description, as follow:

IF Open APP THEN

WHILE $($ Time $>1 \mathrm{~s}$ )//Every 1 seconds down a test real simple (time, resources, small) //judgment for acceleration a information

IF $(3<\Delta a<9)$ THEN //For all test acceleration in changes in the $1 \mathrm{~s}$ in the band has //more than 33 peaks rather than one-way incremental divided into wrestling-band low-pass filter " 3 " //in order to filter out high frequency component in the signal, eliminate or weaken some of the signal //varies, so as to achieve the effect of noise reduction to carefully judge.

The acceleration $a$, decomposition of the body coordinates the acceleration w, model in the direction of supporting human motion, judgment fell to send a message to the SMS module, and recorded in the file.

EISE Record information in the file

END IF

\section{END WHILE}

In judgment fall module after judgment has been falling, and the phone will automatically obtain the longitude and latitude of the location information, and associated API access to specific locations, will coordinate edits to text messages. The system automatically writes information, specific location information including tips for fall, and falls [4]. After countdown cycle, system setting the countdown time (one minute). In a matter of minutes, "cancel sending" button is clicked, information cancel sending. After the end of the countdown, the message will be sent. In a minute, if the old man does not fall down or not serious, you can manually cancel sending, so as to avoid the family unnecessary worry. According to the implementation process, SMS module can be broken down into: GPS data acquisition module, the associated map API module, module to send location information. Data flow diagram in Fig 4.

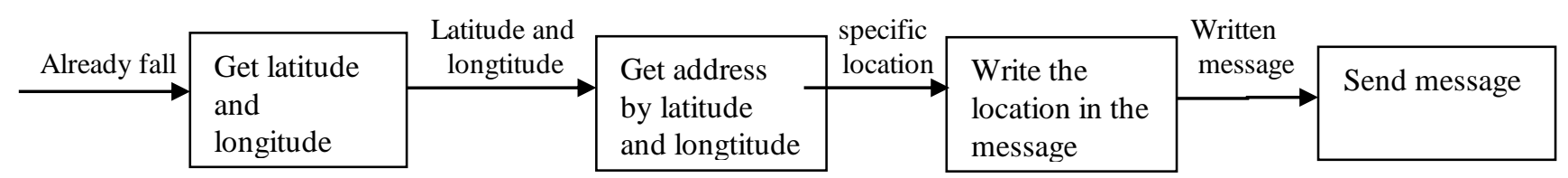

Fig 4. For help send message

GPS information obtaining module is responsible for obtaining the phone's latitude and longitude coordinates, using a Location Manager, gets fixed by specifying Location Provider information, location information represented by the Location object. After the Location Manager object is created, call Location Manager get Provider () method to get a Location Provider. Setting changes the listener gets positioning information from a Location object. The latitude and longitude data as an output stream input to the associated map API module $b$. When the associated map API modules to obtain the latitude and longitude information, through AMAP class guide map development kit comes with specific location information [5]. For more information needs to be registered as a guide map developer and application secret key. And then you need to download the Android Development Kit and are installed on the specified project directory. This location information as sending information input. c. Send information once the module gets the position coordinates, location information will be included in the 
message. Text messaging in addition to location information is compiled in advance. The sending SMS algorithm pseudo code description, as follow:

IF already fall THEN

Get latitude and longitude and encapsulated in a Location object

Created GeocodeQuery (GeocodeSearch) object to a LatLonPoint object

LatLonPoint object parsing function is called to obtain location information

Specific location information generated by SMS

WHILE Countdown $>0$ DO

IF Cancel the Send button is pressed THEN

Cancel message sending

End transmission module features

END IF

Countdown to minus one second

END WHILE

Send a message!

END IF

\section{Results and analysis}

The programmers take downloads from the complete division of app acceleration code testing app phone gets and log files to 5 groups to speed up testing, 5 forward speed test, test 5 group fell, recording and acceleration of changes draw a line chart, and a set of fall and acceleration of three dimensional coordinate decomposition drawing comparisons, analyze acceleration changes in fall

In Figure 5,6,7, as shown acceleration, running is a one-way, slow running and Acceleration steady transformation, and acceleration of the fall is sudden changes in a short time, and at least three peaks, there is a clear distinction between the three, so we designed by acceleration change judgment is reasoned to fall. Velocity has a direction vector, and acceleration values fluctuate, XYZ direction description decomposition acceleration increases, a decrease. Observed by XYZ fell acceleration diagram, $\mathrm{x}$ rises, $\mathrm{y}$ was flat or falling, and $\mathrm{z}$ declined.

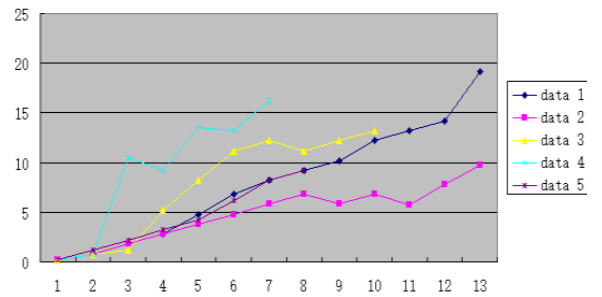

Fig 5. Accelerated mutation testing

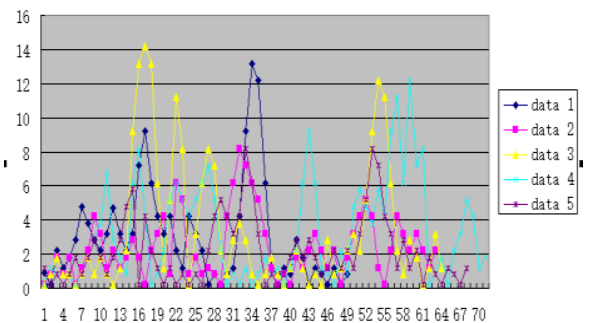

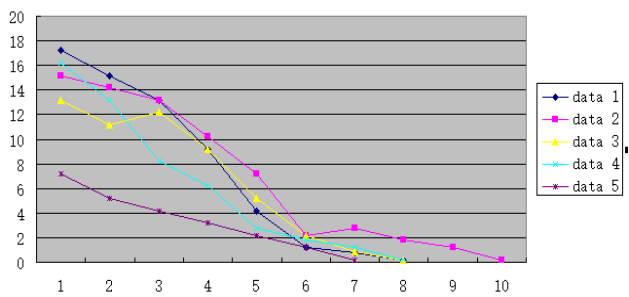

Fig 6. Speed test

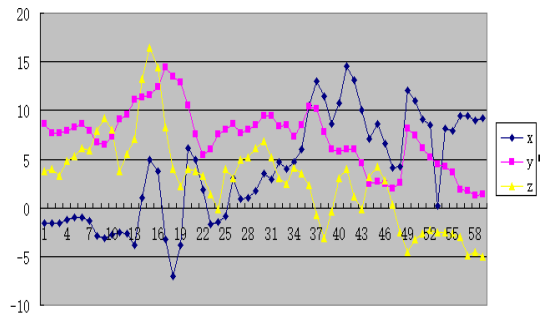

Fig 7. Fall test

Fig 8. Decomposition of mutation testing

Carrying downloads from the complete app divided into GPS code testing app phone Gets and log files, designed routes at Jilin University campus for a week, records GPS latitude and longitude of a scatter graph, and compared with the Jilin University plans route the drawing, testing mobile phone gets GPS accuracy, as shown in Fig.9 and Fig.10. 


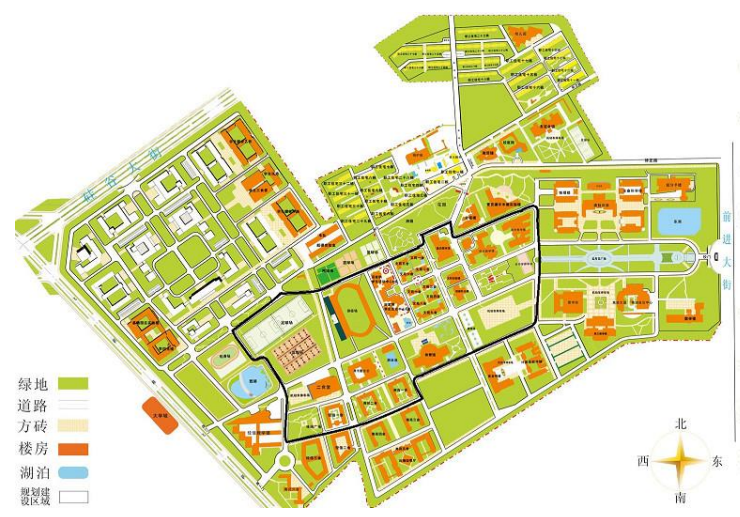

Fig 9. Test route

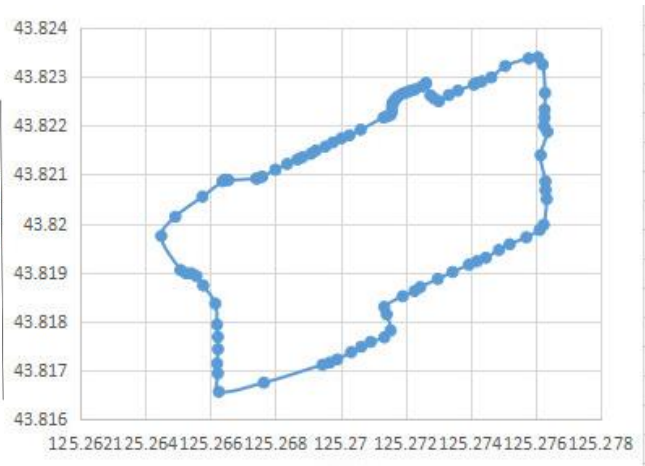

Fig 10. GPS of a scatter graph

\section{Conclusion}

Introduced fell judging app designed for the elderly based on the Android platform. The APP through the human body dynamics model achieves gets old. Use cell phone GPS to obtain location, read the mobile contacts to achieve a message sent. The APP uses the intelligent design, just the initial selected contacts, and automatically without the need for redundant operation for the elderly in falls for help. The APP in order to ensure the safety of life and health of the elderly play a role.

\section{Acknowledgements}

This study of the paper was supported by natural science foundation of Jilin province (2017 0101133JC), innovation and entrepreneurship training program for college students of Jilin University (2016B54512).

\section{References}

[1] Hua, JY ,Shen, ZY , ;Zhong, S , Shen, ZY ,Zhong, S . We Can Track You if You Take the Metro: Tracking Metro Riders Using Accelerometers on Smartphones.IEEE TRANSACTIONS ON INFORMATION FORENSICS AND SECURITY .Vol. 12(2017) No.2 ,p.286-297

[2] Fras, M ,Bednarz, M. Simple Rule-Based Human Activity Detection with Use of Mobile Phone Sensors37th International Conference on Information Systems Architecture and Technology (ISAT) .Karpacz, POLAND. SEP 18-20, 2016 ,p. 39-49

[3] Li, Jing; Song, Ningfang; Yang, Gongliu. Improving positioning accuracy of vehicular navigation system during GPS outages utilizing ensemble learning algorithm.INFORMATION FUSION . Vol. (2017) No.59, p. 1-10

[4] Zheng, Weimin; Huang, Xiaoting; Li, YuanT. Understanding the tourist mobility using GPS: Where is the next place?OURISM MANAGEMENT Vol. (2017) .No.59 .p.267-280

[5] Wang, Ping; Zhang, Yangyang; Hong, Yang.Design of Elderly Tumble Detection System Based on Fuzzy Algorithm. 6th International Symposium on Computational Intelligence and Design (ISCID) .Hangzhou, PEOPLES R CHINA . OCT 28-29, 2013 ,p.169-172 